\title{
Use of Platelet-Rich Plasma for Collagen Matrixes Revitalization with Human Fibroblast
}

\author{
Maxim Sergeevich Makarov*, Natalya Valerjevna Borovkova, Olga Ivanovna Konushko, \\ Valery Borisovich Khvatov \\ Scientific Emergency Aim Institute of N. V. Sklifosovsky, Health Department of Moscow, Moscow, Russia \\ Email: ${ }^{*}$ mcsimmc@yandex.ru
}

Received 15 September 2015; accepted 11 October 2015; published 14 October 2015

Copyright (C) 2015 by authors and Scientific Research Publishing Inc.

This work is licensed under the Creative Commons Attribution International License (CC BY).

http://creativecommons.org/licenses/by/4.0/

c) (i) Open Access

\begin{abstract}
We studied in vitro attachment, proliferation and survival of cadaver skin fibroblasts in collagen bands, dermal matrix and cancellous demineralized bone, enriched with platelet components. It was shown, that PRP enhanced revitalization of collagen grafts, especially followed by components of activated or freezed platelets. Fibroblasts had the best rate of proliferative activity in response to $150 \mathrm{pg}$ platelet derived growth factor (PDGF), released from 100 million platelets with granules and measured for $100 \times 10^{3}$ cultivated cells. The use of platelet-derived material could increase fibroblast proliferation activity for 1.5 - 3 times in all types of collagen transplants without damage or decay of cell's biological value.
\end{abstract}

\section{Keywords}

Platelets, Fibroblasts, Adhesion, Proliferation

\section{Introduction}

Collagen matrixes are widely used in cell biology, including studies of proliferative activity, adhesion, locomotion of the cells [1]-[6]; 3-D matrixes allow to process in vitro tissue remodeling and biological grafts revitalization [2]-[5]. It was shown, that collagen-based biological grafts, combined with cultivating cells, enhance efficiency of clinic care in traumatology, surgery, sport medicine rather than collagen matrixes without cells [7]. On the other hand, the procedure of collagen graft revitalization could be complicated by many factors, including density and topographical features of the graft [1] [3] [5], low locomotive and proliferative cell activity, cell damage [4]-[7]. The bio-engineering methods devise various types of cell settlement inside matrix, and the one

${ }^{*}$ Corresponding author.

How to cite this paper: Makarov, M.S., Borovkova, N.V., Konushko, O.I. and Khvatov, V.B. (2015) Use of Platelet-Rich Plasma for Collagen Matrixes Revitalization with Human Fibroblast. Journal of Biosciences and Medicines, 3, 80-87.

http://dx.doi.org/10.4236/jbm.2015.310011 
way is to use fluids with platelet components—blood serum, fibrinolytic active plasma, platelet-rich plasma [7] [8] [10] [13] — due to unique biological properties of platelets, containing the vast amount of growth and other factors, commonly involved in many processes of reparation and regeneration [9]. But the use of PRP for healing process might be abrogated by hardly predisposed negative effects, such as excessive synthesis of collagen, scar tissue formation, transformation and death of cells [12]-[14]. Noticeably that studying the role of PRP-bearing transplants in clinic care, most of researches did not elucidate the quality of the platelets source, besides their number per ml. Thus, manufacturing of combined transplants with human platelets should be optimized, especially in case of reduction of cell failures.

The aim of this work is to study the biological effect of platelet components in collagen matrixes revitalization, applied in clinical practice.

\section{Materials and Methods}

\subsection{Collagen Matrixes Preparation}

We used collagen bands with human type 1 collagen, performed for burns wound treatment [7], devitalized dermal matrix, harvested from cadaver skin [13], and cancellous demineralized bone, CDB [14]. Each of the studied transplants was cut under sterile conditions into 1.0 - $1.5 \mathrm{~cm}$ slices and placed in the control and experiment Petri dishes. Evaluation of the collagen fibers topography and the density of their packaging maintained study of collagen autofluorescence, using fluorescent lamp "Nikon C-HGFi" $\left(\lambda_{\text {excitation }}=380-420 \mathrm{~nm}\right.$, $\lambda_{\text {emission }}-$ from $450 \mathrm{~nm}$, exposure time- $1 \mathrm{~s}$ ) with further analysis of images in confocal microscope "Nikon Eclipse 80i" (Japan). Autofluorescence intensity of individual collagen fiber was evaluated in ft-candelas by method of vital-stained cells observation [15].

\subsection{PRP Preparation}

Platelet-rich plasma (PRP) was received from donor blood, preserved on citrate, after centrifugation at $350 \mathrm{~g}$ for $5 \mathrm{~min}$. Using the method of assessing the functional status of human platelets [16], we counted the number of platelet with granules in PRP and prepared aliquot doses with 50 - 200 million platelets with granules. In experiments we used samples of unactivated PRP; PRP, activated by liquid collagen ( $2 \mu \mathrm{g} / \mathrm{ml})$ or $10 \%$ calcium chloride $(30 \mu \mathrm{l} / \mathrm{ml})$; PRP after 2 hours of freezing platelets in $-20^{\circ} \mathrm{C}--40^{\circ} \mathrm{C}$ (cryoPRP). Activated PRP and cryoPRP were centrifuged at $4000 \mathrm{~g}$ for $10 \mathrm{~min}$ to eliminate platelets and their fragments. Then prepared doses of PRP were put into Petri dishes with collagen matrixes immediately before cells passage, after 3 and 6 days of cultivation. Saturation platelet materials with growth factors carried out of the content of the platelet growth factor (PDGF-BB). Concentration of PDGF-BB was determined by using a set of reagents "Qantikine, Human PDGF-BB Immunoassay” firm “R(D) \& Systems” and "system Multiskan” ascent company "Thermo” (USA).

\subsection{Cell Culture}

Primary cultures of cadaver skin fibroblasts (CSF) obtained under the standard scheme from tissue donors [17]. Skin fibroblasts are considered to be one of the best cell cultures to study the interaction of cells with collagen matrixes [1]-[4]. CSF were cultivated in DMEM medium with the addition of $10 \%$ fetal bovine serum (Gibco, Russia) on the 6th well firm "Costar" at $37^{\circ} \mathrm{C}$ and $5 \% \mathrm{CO}_{2}$. Cells were separated from the bottom of the bottle with trypsin-Versen, resuspended and put in Petri dishes at the rate of $100 \times 10^{3}$ cells in 1 well. Change of medium conducted over 3 days, the total time of the cultivation of CSF amounted 7 days.

\subsection{Cell Study}

Cultivated cells were studied with confocal microscope "Nikon Eclipse 80i" (Japan), using our method of vital-stained cells observation [16]. The study of cells in culture and in the grafts determined the following parameters: number of adhesive cells, $10^{3} / \mathrm{cm}^{2}$; proliferation index at third day culturing, Ip3 (defined as the ratio of the number of cells after 3 days of cultivation to the quantity of cells per 1st culturing days), cell's membranes integrity (CMI, in points). Normally, the CMI varies from 28 to 42 points [16]. Received statistical data processed using the variation statistical methods using the software package "Microsoft Excel 2000". Calculate arithmetic values (M) and standard deviation $(\sigma)$. Differences of values considered reliable in more than 95\% significance level. 


\section{Results and Discussion}

Autofluorescence ability is the natural feature of collagen, which allows visualizing the collagen-containing components in fluorescent microscope to assess architecture and density of collagen [18]. In collagen bands autofluorescence intensity level averaged $9.5 \mathrm{ft}$-candelas, in dermal matrix-28.6 ft-candelas, in CDB-85.9 $\mathrm{ft}$-candelas. According to our data, that in raw human skin collagen autofluorescence intensity level is 27 - 30 $\mathrm{ft}$-candelas, in cancellous bone- 80 - $90 \mathrm{ft}$-candelas, it is concluded that original structure of collagen fibers maintained in the dermal matrix and CBD. In this case, in collagen dermal matrix and bands the thickness of individual fibers was similar and the difference in autofluorescence level was determined mainly by the distance between the fibers (Figure 1(a)), whereas CDB samples had very dense branches of fibers with very high autofluorescence intensity (Figure 1(b)). This indicates a fundamental difference between packaging collagen in cancellous demineralized bones comparing to collagen bands and dermal matrix, that led to much less pronounced adhesion of skin fibroblasts on CBD surface after 1 day of cultivation (Figure 2). The registered level of CSF adhesion on the surface collagen bands and dermal matrix was $6 \times 10^{3} / \mathrm{cm}^{2}$ and $\operatorname{InP} 3=1.5$, time of monolayer formation on the surface of these transplants estimated not less than 7 days, i.e. was quite low. Therefore, in order to enhance collagen matrix revitalization cells we used the growth-stimulating factors of human PRP.

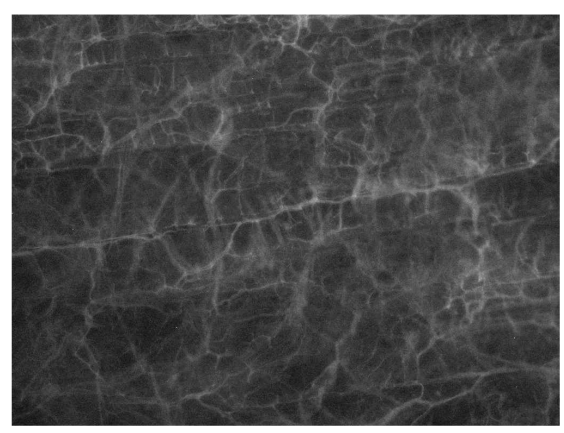

(a)

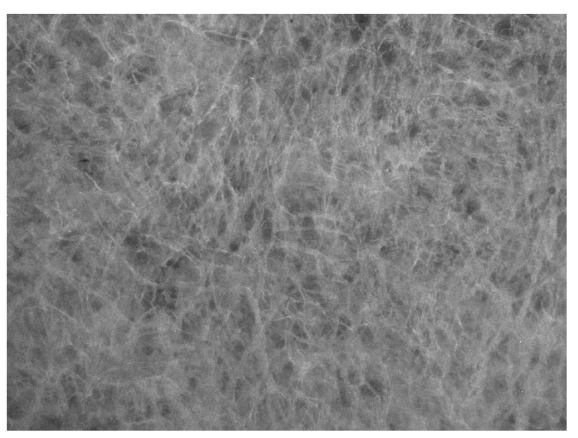

(b)

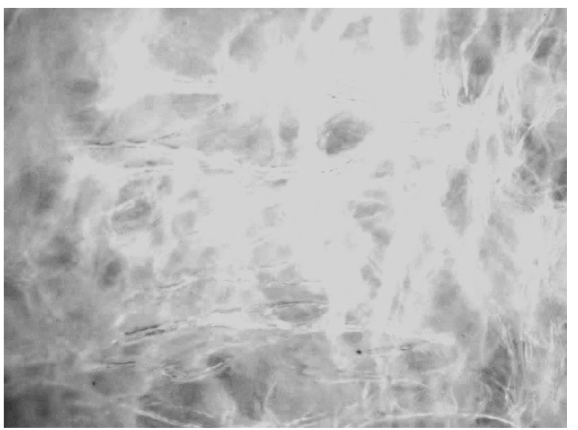

(c)

Figure 1. Collagen autofluorescence in collagen bands (a); dermal matrix (b) and cancellous demineralized bone (c) during 1 day of the experiment. Magnification $\times 50$. 


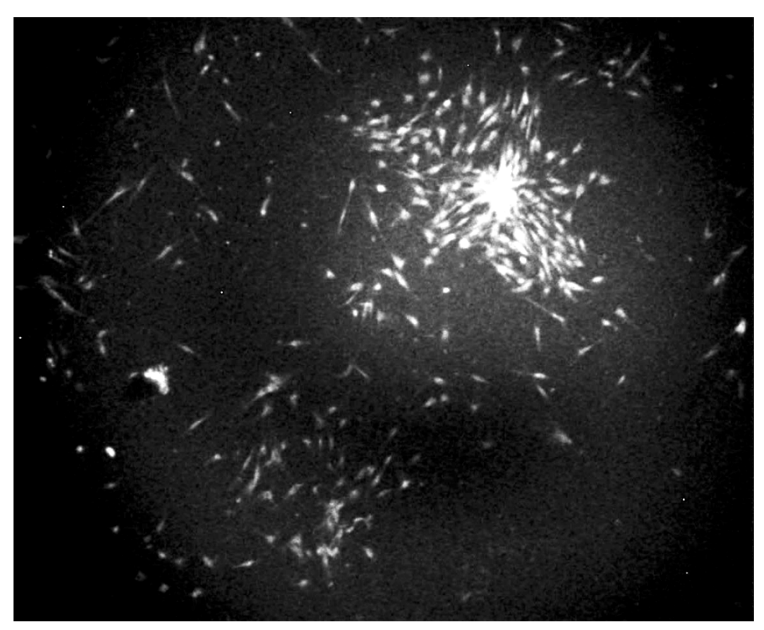

(a)

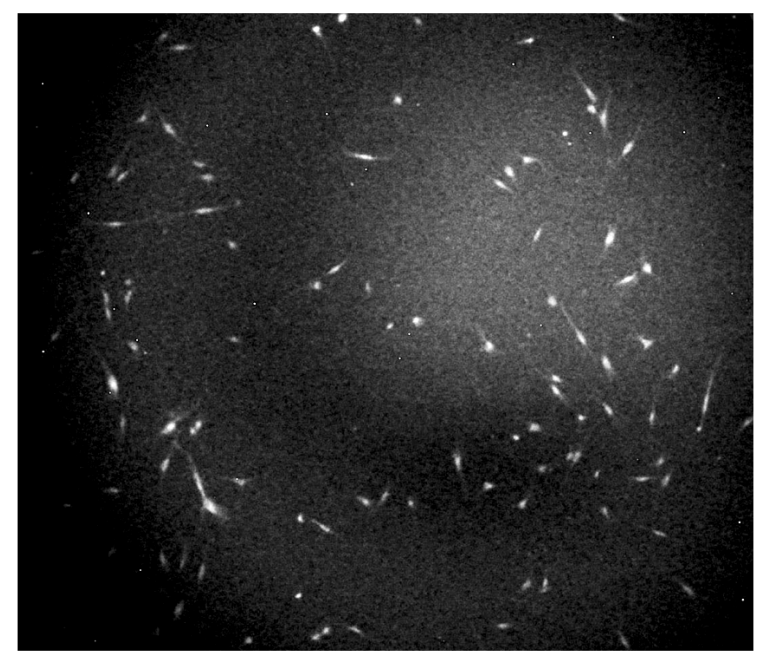

(b)

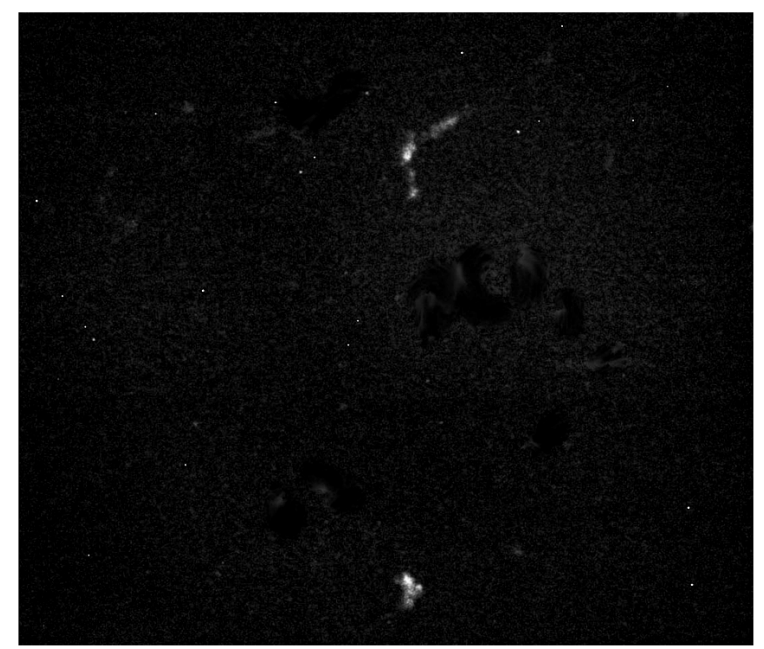

(c)

Figure 2. Cadaver skin fibroblasts, anchored at surface of collagen matrixes during 1 day of the experiment. Vital staining with trypaflavin-rodamin C. (a) Collagen bands; (b) Dermal matrix; (c) Cancellous demineralized bone. Magnification $\times 50$. 
Before applying the PRP it was necessary to determine the optimal dose of platelet components for CSF culture. Our previous study of human fibroblasts $\mathrm{M}-22$ found that the maximum rate of the fibroblasts proliferation observed at concentration 120 - 150 pg PDGF-BB per $100 \times 10^{3}$ cultivated cells without decay of their structural integrity [19]. In this case, 3d-day’s proliferative index (InP3) varied from 3.5 to 4.0, whereas in control wells (without PDGF-BB) it was only 1.5. We established that the PRP saturation with PDGF-BB was closely linked with the amount of platelets with granules, identified by vital staining. According to different studies, PDGF platelet granule contents seemed to be correlated with the content of the other major growth factors [11]-[13], i.e. the assessment of PDGF-BB in PRP could give an integral feature of its growth-stimulating capacity. In examinated PRP samples concentrarion of platelets with granules was on average 200 - 300 million/ml, concentration of growth factor PDGF-BB in 1 dose (100 million platelets with granules) varied from 0 to $1 \mathrm{ng} / \mathrm{ml}$ in unactivated PRP, in activated PRP or cryoPRP_-from 132 to 167 pg/mL, averaging 149 pg/mL. Thus, total degranulation of 100 million platelets with granules allowed to obtain the required number of PDGF-BB in a calculation for 1 dose.

Study of proliferative activity in CSF culture under influence of PDGF-BB from PRP showed that 60 pg PDGF-BB for $100 \times 10^{3}$ cells increased Ip3 to $1.7,90 \mathrm{pg}$ - to $2.2,120 \mathrm{pg}$ - to $2.7,150 \mathrm{pg}$ - to $3.0,180 \mathrm{pg}$ - to 2.4, 240 pg-to 1.5, whereas in control wells Ip3 did not exceed 1.4. Cell membranes integrity (CMI) through the 3 culturing days in control and experimental wells with $60-150$ pg PDGF-BB amounted to $36.8 \pm 1.4$ points, with $180 \mathrm{pg}-36.0 \pm 1.7$ points, experience with $240 \mathrm{pg}-32.2 \pm 2.3$ points, comparing to $37.0 \pm 2.5$ points in control. Therefore, the concentration 150 pg PDGF-BB was non-toxic and most effective for stimulation of cell proliferation. In view of the fact that 150 pg PDGF-BB is released from 100 million platelets with granules (biologically high-grade platelets), it confirmed the effectiveness of this dose for $100 \times 10^{3}$ cells in CSF culture.

The next step of the research was saturating of the collagen matrixes with platelets components in vitro to give them growth-stimulating properties. It must be noticed, that the PDGF-BB output, as well as other growth factors, can be stimulated by different ways: 1) platelet degranulation during the process of adhesion; 2) degranulation under the influence of activation inductor; 3) degranulation during clot formation; 4) platelet destruction under the influence of a chemical or physical factors. In PRP-treated collagens bands and dermal matrix settlement of CSF was similar to the one recorded in the control wells. By the end of the 1th day number of cells on the surface of these grafts averaged 6.1 and $5.8 \times 10^{3} / \mathrm{cm}^{2}$. In wells with unactivated PRP one could observe total platelet activation, accompanied by their degranulation, but direct collagen adhesion was observed only in $10 \%$ of the total number of platelets and the remaining 90\% platelets aggregated and effluxed granules in medium without collagen contact. Presence of platelet material did not significantly affect the adhesion of fibroblasts in wells with collagen bands and dermal matrix. In PRP-treated CDB process of cells settling from suspension was expressed weaker: after 1 day of culturing in wells with activated PRP and cryoPRP number of CSF amounted to $(1.3-1.4) \times 10^{3} / \mathrm{cm}^{2}$, in wells with unactivated $\mathrm{PRP}-0.1 \times 10^{3} / \mathrm{cm}^{2}$, i.e. CSF adhesion was the same as in control. It is necessary to emphasize that the platelets did not completely set on the surface of CDB.

After 3 days of CSF cultivation in collagen bands and dermal matrix with the activated PRP and cryoPRP InP3 was on average 1.8 times higher than in control, in the CDB-more than 3 times (Table 1). By this time the surface collagen bands and dermal matrix was formed by confluent monolayer-area occupied by the cells varied from 79 to 88 per cent (Figure 3(a) and Figure 3(b)), while there were no visible structural violations as in cells within collagen matrixes (CMI = 36.5 \pm 1.3 points, Figure 3(a') and Figure 3(b')), and in cells growing

Table 1. Growth of cadaver skin fibroblasts on collagen matrix’s surface.

\begin{tabular}{ccccccc}
\hline \multirow{2}{*}{ Collagen matrix type } & \multicolumn{2}{c}{ Collagen bands } & \multicolumn{2}{c}{ Dermal matrix } & \multicolumn{2}{c}{ Cancellous demineralized bone } \\
\cline { 2 - 7 } & $\begin{array}{c}\text { number of adhesive } \\
\text { cells, } 10^{3} / \mathrm{cm}^{2}\end{array}$ & InP3 & $\begin{array}{c}\text { number of adhesive } \\
\text { cells, } 10^{3} / \mathrm{cm}^{2}\end{array}$ & InP3 & $\begin{array}{c}\text { number of adhesive } \\
\text { cells, } 10^{3} / \mathrm{cm}^{2}\end{array}$ & InP3 \\
\hline control & $9.0 \pm 0.5$ & $1.5 \pm 0.1$ & $7.5 \pm 0,5$ & $1.2 \pm 0.1$ & $0.1 \pm 0.1$ & $1.0 \pm 0.1$ \\
unactivated PRP & $16.2 \pm 1.0^{*}$ & $2.5 \pm 0.2^{*}$ & $11.4 \pm 1.1^{*}$ & $1.9 \pm 0.1^{*}$ & $0.1 \pm 0.1$ & $1.0 \pm 0.2$ \\
activated PRP & $16.8 \pm 1.1^{*}$ & $2.7 \pm 0.2^{*}$ & $13.5 \pm 1.3^{*}$ & $2.2 \pm 0.2^{*}$ & $5.2 \pm 2.2^{*}$ & $3.6 \pm 0.4^{*}$ \\
cryoPRP & $16.7 \pm 1.0^{*}$ & $2.6 \pm 0.4^{*}$ & $13.3 \pm 1.6$ & $2.1 \pm 0.2^{*}$ & $4.9 \pm 1.1^{*}$ & $3.4 \pm 0.3^{*}$ \\
\hline
\end{tabular}

"Comparing to control type at $p<0.05$. 


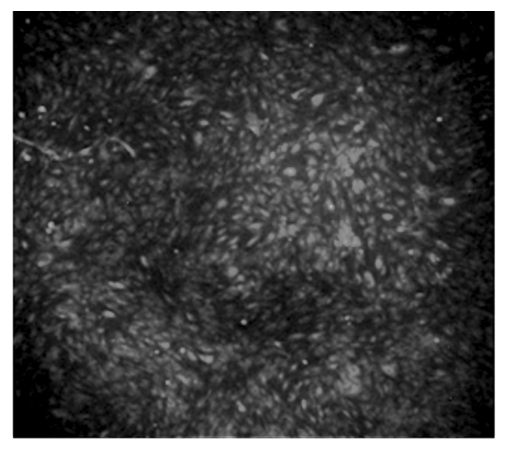

(a)

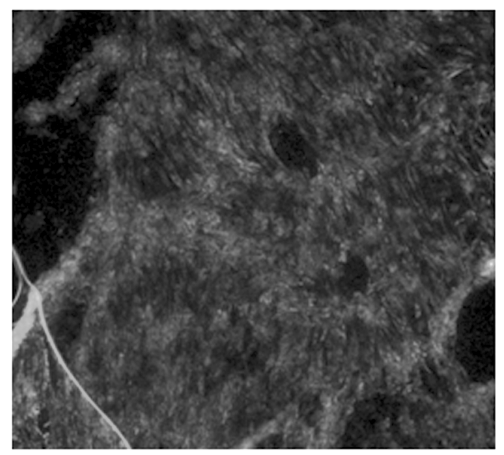

(b)

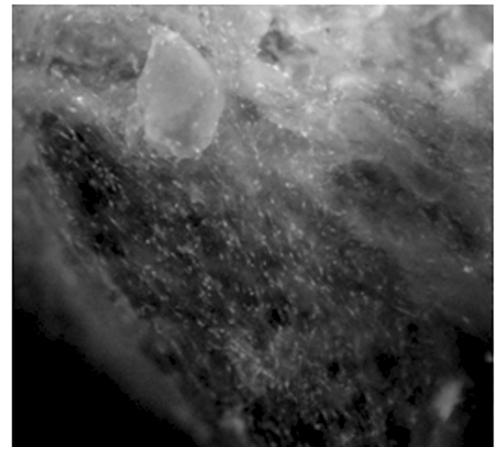

(c)

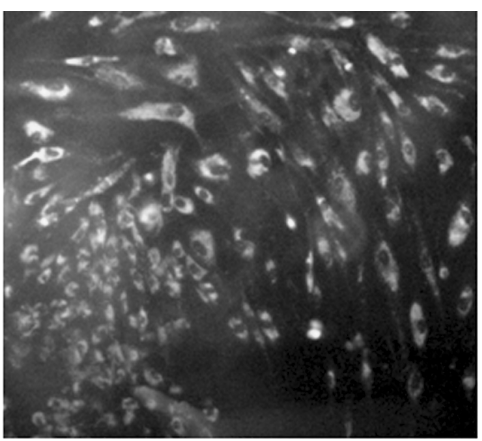

(a')

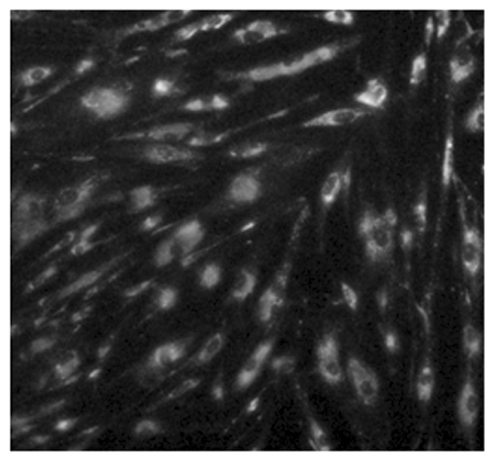

(b')

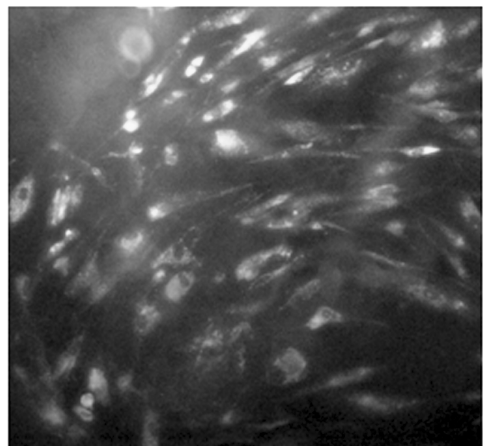

(c')

Figure 3. Monolayer formation on surface of collagen matrixes in presence with platelet material from activated PRP. (a), (a') Collagen bands (3 days of cultivation); (b), (b') Dermal matrix (4 days of cultivation); (c), (c') Cancellous demineralized bone (7 days of cultivation). Magnification $\times 50$ (left column) and $\times 400$ (right column).

on the bottom of the well (CMI $=36.6 \pm 0.9$ points). In experiments with CDB after 3 days monolayer did not form, but the proliferation of cells continued during the later cultivation in the presence of activated PRP or cryoPRP. As a result through 7 - 8 days confluent monolayer was observed on the surface of the CDB, maintaining the structural integrity of the cells (Figure 3(b')). In experiments with unactivated PRP after 3 days of CSF cultivation of collagen bands and dermal matrix Ip3 rate exceeded the similar values in control at 1.5 - 1.6 times. Confluent monolayer formed through 4 days, i.e. the intensity of cells proliferation was lower than in other experiments, but much higher than in control. In experiments with CDB cell did not colonize biotransplant slices (Table 1), cells growth on the bottom of well was equal to control wells. We noticed the rude decline in the number of platelets with granules in the medium - if in the origin PRP their relative content was $60 \%$, after 3 days of experience it was only $17 \%$. Apparently, the gradual destruction of unactivated platelets was not accompanied by release of growth factors in concentrations, sufficient to increase growth of CSF. This confirms the evidence that decay of human platelet's quality during storage at room temperature and constant 
stirring is not associated with the release of granules for their limits, and commonly depends on intracellular processes [19]. The process of dermal matrix revitalization is still not widely described; certain studies on porcine dermal matrix implants in living animals showed quite low migration and proliferative activity of cell during long period [20], also complicated by absence of blood vessel's growth. Density and arrangement of collagen fiber plays dramatic role in fibroblast spreading and proliferation: widely-branched net of thin fibers (like in collagen band) is rather adhesive than lonely thick fibers [1]-[4], so most of collagen matrixes, based on devitalized tissues, remain hardly used in production combined biotransplants, nearby, using standard methods of cell cultivation. Nevertheless, the use of PRP components could noticeably enhance this process, but the ways of platelet incorporation into biotransplants are still discussible. On the one hand, growth factors, released from activated PRP and cryoPRP, had the most positive effect on fibroblast growth inside all types of studied transplants. On the other hand, there is a risk to lose soluted platelet factors, that could be saved in spread platelets, using previously unactivated PRP. So the aim of futher experiments is to stimulate platelets spreading on collagen biotransplants, arranged of dense fibers. The lack of platelets adhesion on the surface of the CDB may be proposed by inability of collagen binding centers to contact platelet integrins, hindered by density of matrix or non-collagen proteins in the bone [3]. This factor can also be a cause of low adhesion of cultured cells on the surface of the CDB. However, processing of activated PRP or cryoPRP helps relieve this problem; apparently, this effect is caused by partial deformation of dense collagen fibrils under action of lysosomal hydrolases and other factors from platelet granules.

Fibroblast migration in the internal area of collagen matrixes, saturated with platelet components during the whole period of cultivation was quite low and occured only through the activity of cells surface monolayer. After 7 days of cultivation maximum penetration depth of CSF samples in dermal matrix amounted to $20 \mu \mathrm{m}$, in $\mathrm{CDB}-10 \mu \mathrm{m}$, and did not depend on way of platelets processing. One could conclude, that total revitalization of biotransplants could estimate more than 30 days.

The efficacy of acellular dermal matrix was shown during treatment of full-thickness skin injuries [13], but revitalization of this matrix in vivo had been poorly studied yet, mainly, due to evoking of inflammatory and immune reaction [10]. Nevertheless, in vitro colonization of dermal matrix with allogenic cells is potentially reliable; therefore, it could be performed also with autological cells in measurement with PRP components. We suspect, that thin collagen matrixes with human PRP could be both biotransplants and healing band, especially actual for cut and burn wound treatment, what might to be the aim of futher investigations. Thick collagen trasnplants, with dense packaging of fibers seem to be ineffective substrate for platelet adhesion; therefore, the manufacturing of bone grafts with high-value platelets needs previous bone covering with platelet-attractive material-as a variant, collagen, hyaluronic acid, chitosan, different types of carbon polymers could be used. According to interest in both bone grafts and PRP in traumatology, combination of these materials really worth studying. The exactly checked dose of PRP can obviously enhance the bone remodeling.

\section{Conclusion}

Saturation of collagen matrixes with platelet components stimulated human fibroblast proliferation, undergone by platelet growth-stimulating factors output. Otherwise, it is shown that prolonged use of PRP in platelet quality-reducing conditions led to decay of reparative and regenerating effect. This points both importance of matrix preparation procedure and assessment of platelet biological integrity [8]. In addition, the concentration of the platelets components also played a dramatic role. Dose of growth-stimulating factors, extracted from 100 million platelets with granules, appears to be the most effective and non-toxic for cultured cells. On the other hand, it should be noted that in most cases, the cells settlement of collagen matrixes was observed mainly on the surface grafts and depth no more 10 - 20 microns. Deeper penetration of cells failed to register; however, the migration of fibroblast into the interior zones was observed till the 7 days of cultivation. It can be assumed that the full settlement of three-dimensional collagen matrixes requires longer periods of cultivation. The use of biologically high-grade platelets material can significantly enhance the settling of cells in collagen matrices.

\section{References}

[1] Chandran, P.L. and Barocas, V.H. (2006) Affine versus Non-Affine Fibril Kinematics in Collagen Networks: Theoretical Studies of Network Behavior. Journal of Biomechanical Engineering, 128, 259-270.

http://dx.doi.org/10.1115/1.2165699 
[2] Ahlfors, J.E. and Billiar, K.L. (2007) Biomechanical and Biochemical Characteristics of a Human Fibroblast Produced and Remodeled Matrix. Biomaterials, 28, 2183-2191. http://dx.doi.org/10.1016/j.biomaterials.2006.12.030

[3] Friedl, P. and Brocker, E.B. (2000) The Biology of Cell Locomotion within Three-Dimensional Extracellular Matrix. Cellular and Molecular Life Sciences CMLS, 57, 41-64. http://dx.doi.org/10.1007/s000180050498

[4] Jiang, H., Rhee, S., Ho, C.-H. and Grinnell, F. (2008) Distinguishing Fibroblast Promigratory and Procontractile Growth Factor Environments in 3D Collagen Matrices. The FASEB Journal, 22, 2151-2160. http://dx.doi.org/10.1096/fj.07-097014

[5] Pedersen, J.A., Boschetti, F. and Swartz, M.A. (2007) Effects of Extracellular Fiber Architecture on Cell Membrane Shear Stress in a 3D Fibrous Matrix. Journal of Biomechanics, 40, 1484-1492. http://dx.doi.org/10.1016/j.jbiomech.2006.06.023

[6] Grinnell, F. (2003) Fibroblast Biology in Three-Dimensional Collagen Matrices. Trends in Cell Biology, 13, $264-269$. http://dx.doi.org/10.1016/S0962-8924(03)00057-6

[7] Anitua, E., Andia, I., Ardanza, B., Nurden, P. and Nurden, A.T. (2004) Autologousplatelets as a Source of Proteins for Healing and Tissue Regeneration. Thromb Haemost, 91, 4-15.

[8] Mazurov, A.V. (2011) Physiology and Pathology of Platelets. Litterra, Moscow.

[9] Toit, D.F., Kleintjes, W.G. and Otto, M.J. (2007) Soft and Hard-Tissue Augmentation with Platelet-Rich Plasma: Tissue Culture Dynamics, Regeneration and Molecular Biology Perspective. International Journal of Shoulder Surgery, 1, 64-73. http://dx.doi.org/10.4103/0973-6042.32923

[10] Dees, C., Akhmetshina, A., Zerr, P. Reich, N., Palumbo, K., Horn, A., Jüngel, A., Beyer, C., Krönke, G. and Zwerina, J. (2011) Platelet-Derived Serotonin Links Vascular Disease and Tissue Fibrosis. Journal of Experimental Medicine, 208, 961-972. http://dx.doi.org/10.1084/jem.20101629

[11] Wang, Z., Ahmad, A., Li, Y., Kong, D., Azmi, A.S., Banerjee, S. and Sarkar, F.H. (2010) Emerging Roles of PDGF-D Signaling Pathway in Tumor Development and Progression. Biochimica et Biophysica Acta, 1806, 122-130. http://dx.doi.org/10.1016/j.bbcan.2010.04.003

[12] Harrison, P. and Mumford, A. (2009) Screening Tests of Platelet Function: Update on Their Appropriate Uses for Diagnostic Testing. Seminars in Thrombosis and Hemostasis, 35, 150-157. http://dx.doi.org/10.1055/s-0029-1220323

[13] Khubutia, M.Sh., Andreev, Yu.V., Borovkova, N.V., Khvatov, V.B., Mironov, A.S., Zirkova, E.A., Ponomarev, I.N. and Makarov M.S. (2014) Method of Dermal Matrix Production. Patent RU 2,524,619. A61K35/36, A61L27/36.

[14] Bykov, V.A., Denisov-Nikolsky, Yu.I., Denisova, L.A., Matvejchuk, I.V. and Rozanov, V.V. (2006) Method of Bone Implant Production. Patent RU 2,268,060. A61K35/32, A01N1/00.

[15] Makarov, M.S., Khvatov, V.B., Konushko, O.I., Storogeva, M.V., Borovkova, N.V. and Ponomarev, I.N. (2013) Method of Morphofunctional Analysis of Biotransplant Cell Components. Patent RU 2,484,472. G01N33/48.

[16] Makarov, M.S., Kobzeva, E.N., Vysochin, I.V., Borovkova, N.V. and Khvatov, V.B. (2014) Morphofunctional Analysis of Human Platelets by Vital Staining. Bulletin of Experimental Biology and Medicine, 156, 409-412. http://dx.doi.org/10.1007/s10517-014-2360-0

[17] Richards-Kortum, R. and Sevick-Muraca, E.M. (1996) Quantitative Optical Spectroscopy for Tissue Diagnosis. Annual Review of Physical Chemistry, 47, 555-606. http://dx.doi.org/10.1146/annurev.physchem.47.1.555

[18] Makarov, M.S., Storogeva, M.V., Konushko, O.I., Borovkova, N.V. and Khvatov, V.B. (2013) Effect of Concentration of Platelet-Derived Growth Factor on Proliferative Activity of Human Fibroblasts. Bulletin of Experimental Biology and Medicine, 155, 576-580. http://dx.doi.org/10.1007/s10517-013-2199-9

[19] Schubert, P. and Devine, D.V. (2010) Towards Targeting Platelet Storage Lesion-Related Signaling Pathways. Blood Transfus, 8, 69-72.

[20] Monteiro, G.A., Rodriguez, N.L., Delossantos, A.I. and Wagner, C.T. (2013) Short-Term in Vivo Biological and Mechanical Remodeling of Porcine Acellular Dermal Matrices. Journal of Tissue Engineering, 4, 2041731413490182. http://dx.doi.org/10.1177/2041731413490182

\section{Abbreviations}

PRP—platelet-rich plasma; CDB—cancellous demineralized bone; InP3—3d-day’s proliferative index; CSF— cadaver skin fibroblasts; CMI—cell's membrane integrity; cryoPRP—PRP after cryodestruction; PDGFplatelet derived growth factor. 